\title{
Supplimentary Information: The effects of lattice strain, dislocations, and microstructure on the transport properties of YSZ films
}

\author{
George F. Harrington, ${ }^{* a \ddagger}$ Andrea Cavallaro, ${ }^{a}$ David W. McComb,${ }^{b, a}$
}

Stephen J. Skinner, ${ }^{a}$ and John A. Kilner ${ }^{a, c}$

${ }^{a}$ Department of Materials, Imperial College London, London, SW7 2AZ, UK.

${ }^{b}$ Department of Materials Science and Engineering, The Ohio State University, Columbus, USA.

${ }^{c}$ International Institute for Carbon-Neutral Energy Research (WPI-I2CNER), Kyushu University, Fukuoka, Japan.

$¥$ Present affiliations: Next-Generation Fuel Cell Research Center, Kyushu University, Fukuoka, 819-0395, Japan, and Department of Materials Science and Engineering, Massachusetts Institute of Technology, Cambridge, MA 02139, USA.

* E-mail: harrington.frederick.george.302@m.kyushu-u.ac.jp 


\section{PLD target characterisation}

Prior to deposition, the 8 mol.\% YSZ sintered target was measured by x-ray diffraction (XRD) in Bragg-Brentano geometry (PANalytical X'Pert Pro MRD). The $2 \theta / \omega$ scan is shown in Fig. 1. The pattern was indexed as a cubic fluorite structure and showed no signs of any additional phases. The lattice parameter, as extracted from a Le Bail refinement in the FullProf software suite [1], was found to be $0.5139 \mathrm{~nm}$.

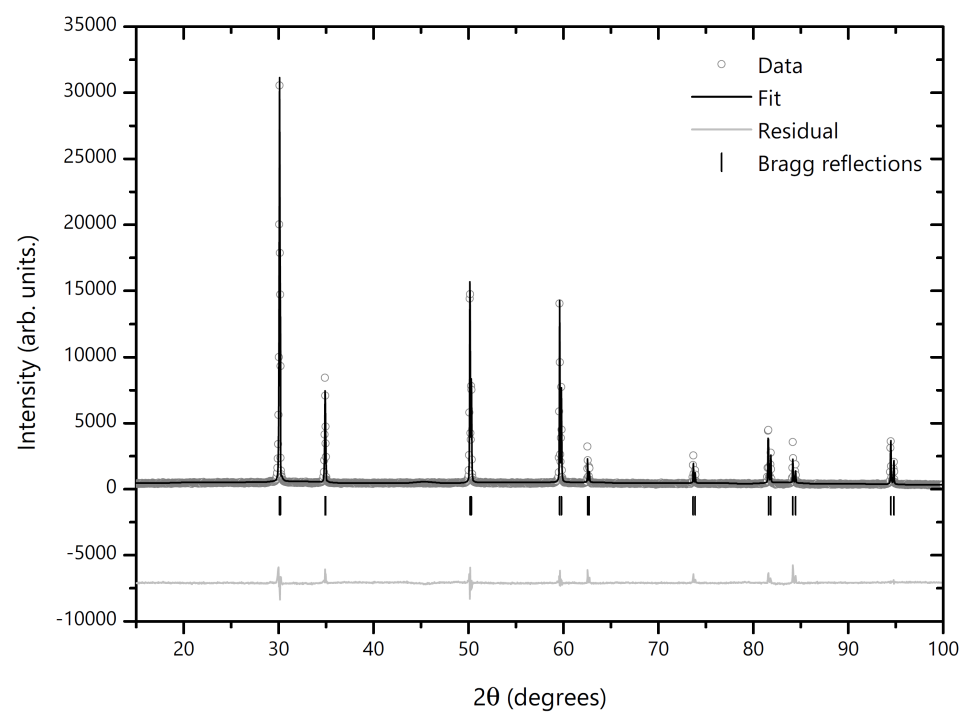

Figure 1: XRD $\theta / 2 \theta$ pattern from the sintered YSZ PLD target prior to deposition. The pattern has been refined using the Le Bail method for a cubic fluorite structure yield a lattice parameter of $a_{Y S Z}=$ $0.5139 \mathrm{~nm}$ 


\section{XRD film characterisation}

\subsection{Out-of-plane measurements}

Prior to the $2 \theta / \omega$ scan acquisition the $2 \theta$ and $\omega$ angles were set to $0^{\circ}$ in order to measure and correct the zero-position of the diffractometer. The lattice parameters of the films were calculated from the $2 \theta$ peak positions. First the substrate peak positions were refined using the Le Bail analysis in the FullProf software, in order to account for any possible errors in the $2 \theta=0$ position. Next the film peaks were fitted using pseudo-Voigt functions in order to calculate the lattice parameters.

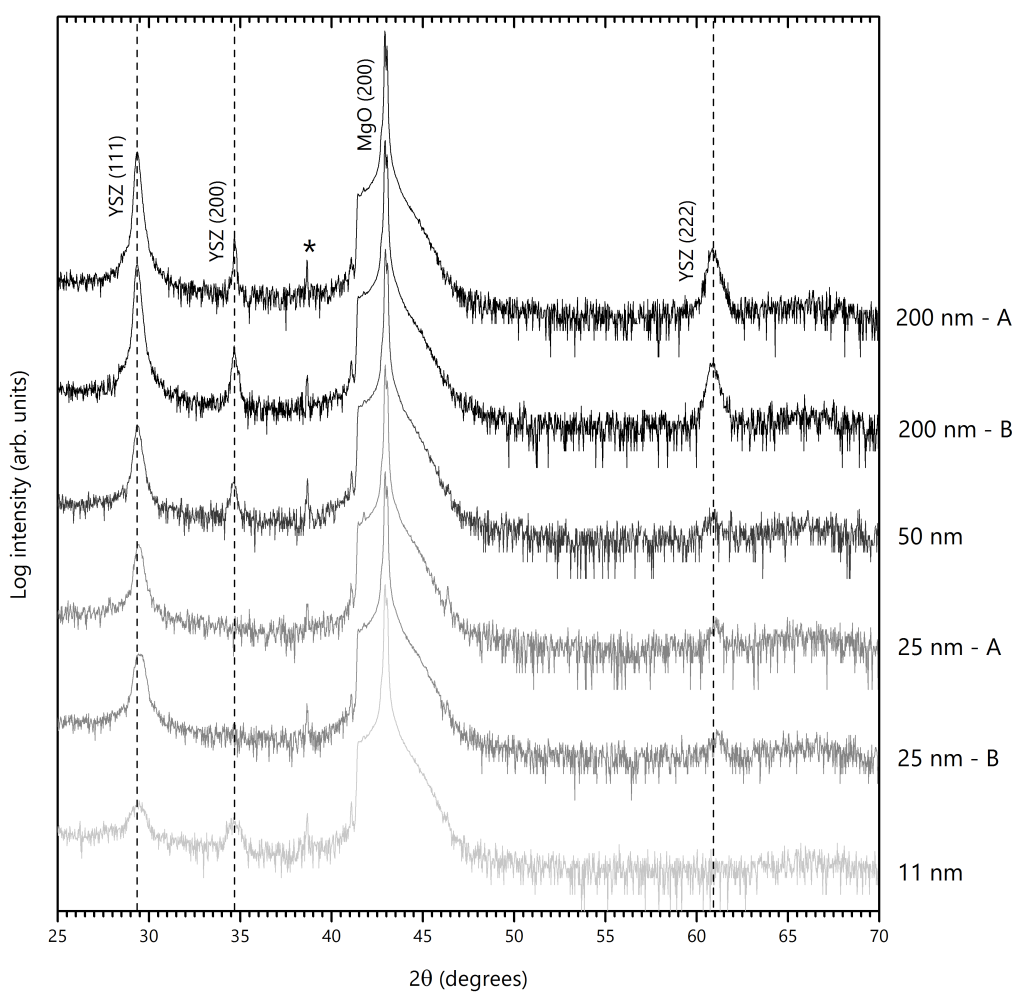

Figure 2: XRD $\theta / 2 \theta$ patterns from the YSZ films grown on $\mathrm{MgO}$ substrates with a nominal substrate temperature of $700^{\circ} \mathrm{C}$ during deposition. The peak marked with a $*$ are due to the substrate peaks. The samples labels correspond to those in Table 1. 


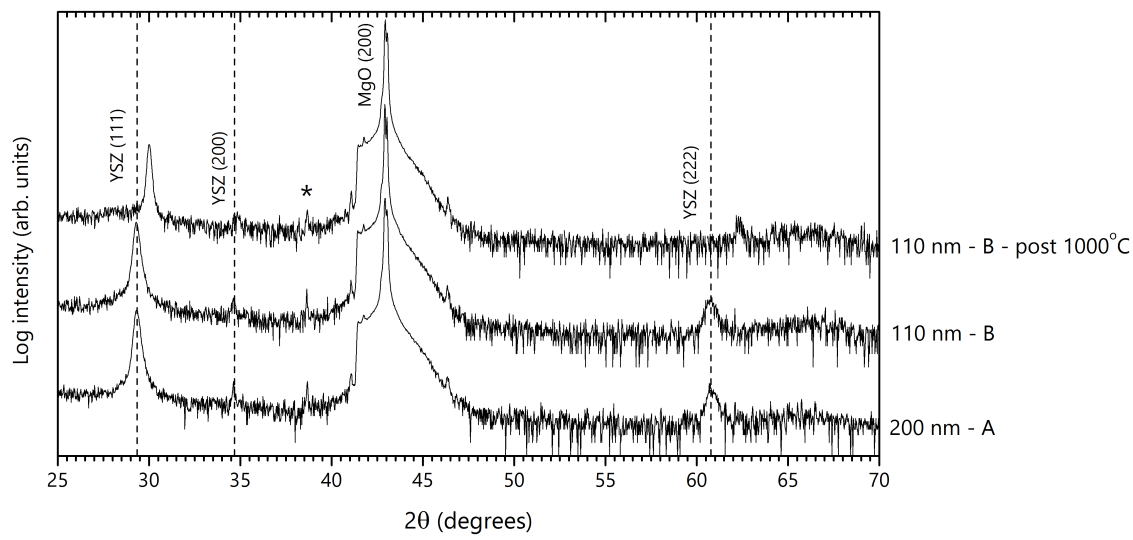

Figure 3: XRD $\theta / 2 \theta$ patterns from the YSZ films grown on $\mathrm{MgO}$ substrates with a nominal substrate temperature of $600^{\circ} \mathrm{C}$ during deposition. The peak marked with a $*$ are due to the substrate peaks. The samples labels correspond to those in Table 1. 


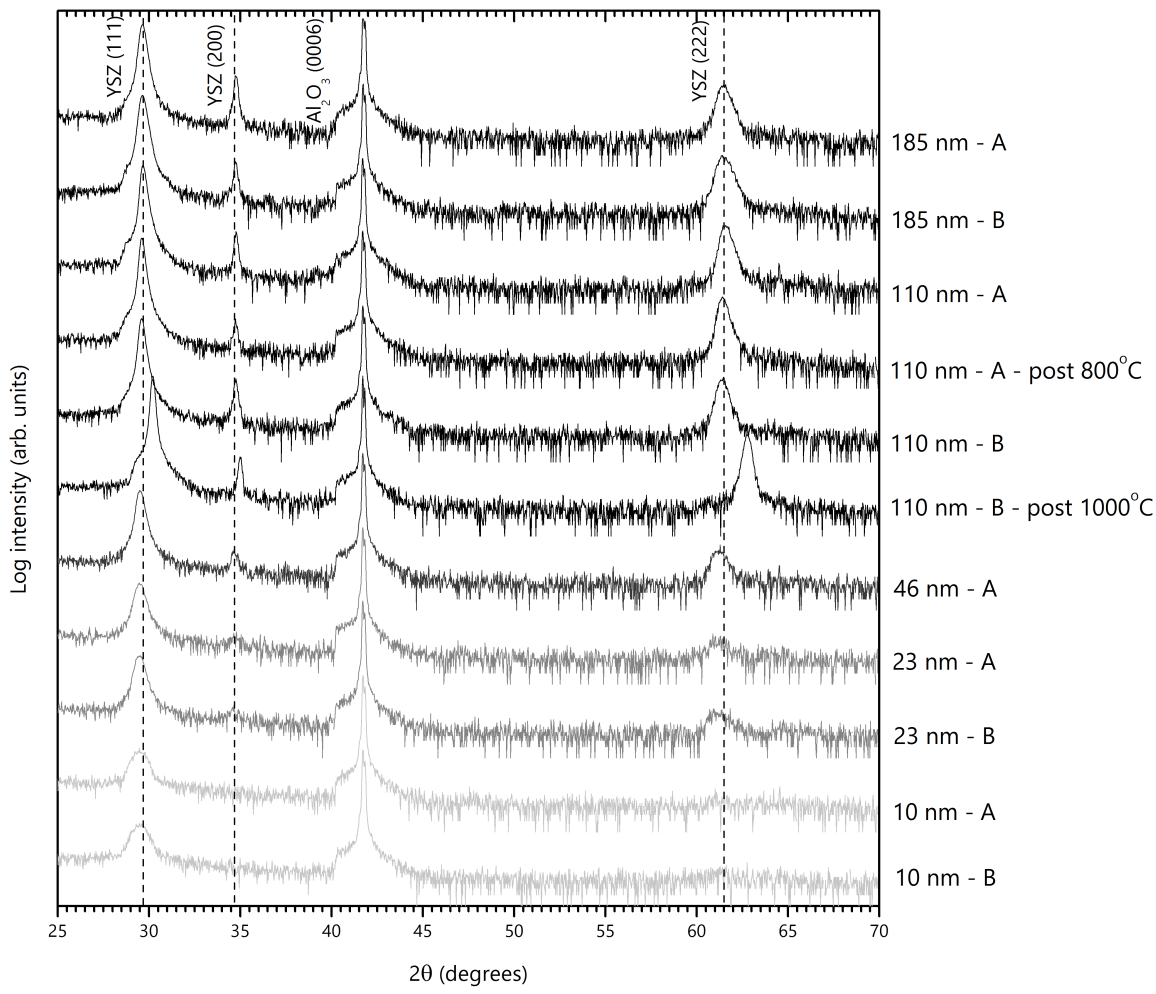

Figure 4: XRD $\theta / 2 \theta$ patterns from the YSZ films grown on $\mathrm{Al}_{2} \mathrm{O}_{3}$ substrates with a nominal substrate temperature of $600^{\circ} \mathrm{C}$ during deposition. The peak marked with a $*$ are due to the substrate peaks. The samples labels correspond to those in Table 1. 


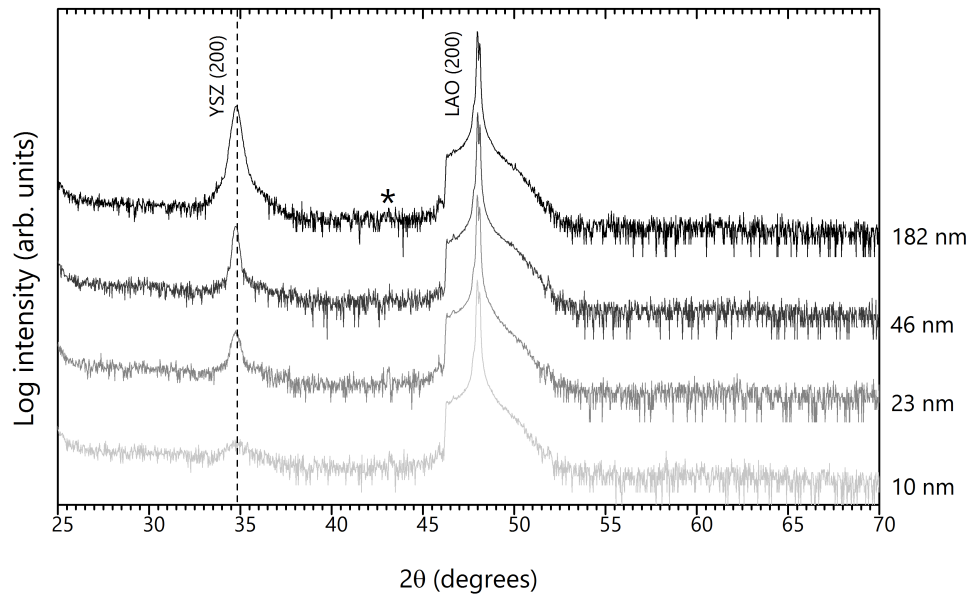

Figure 5: XRD $\theta / 2 \theta$ patterns from the YSZ films grown on LAO substrates with a nominal substrate temperature of $600^{\circ} \mathrm{C}$ during deposition. The peak marked with a $*$ are due to the substrate peaks.

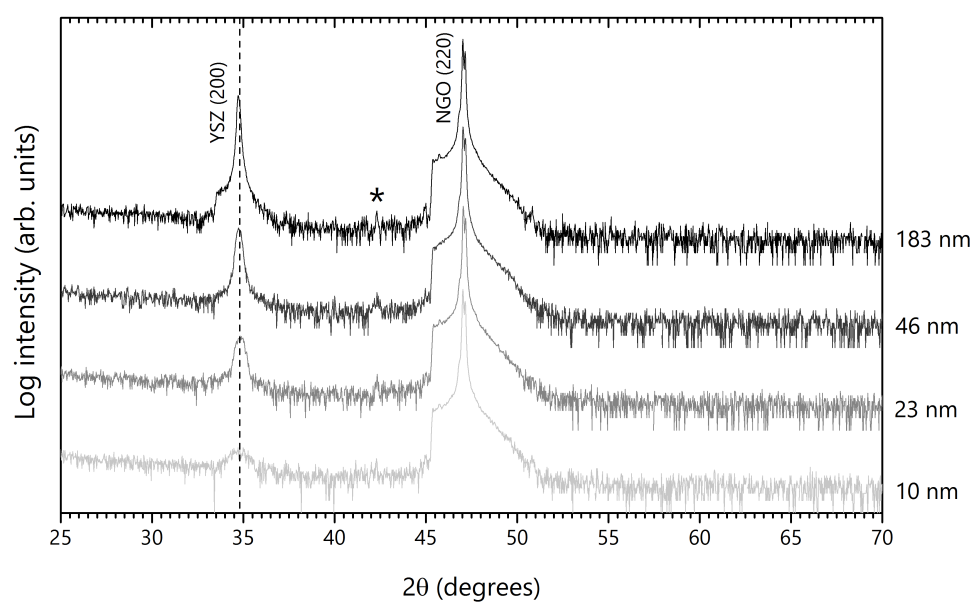

Figure 6: XRD $\theta / 2 \theta$ patterns from the YSZ films grown on NGO substrates with a nominal substrate temperature of $600^{\circ} \mathrm{C}$ during deposition. The peak marked with a $*$ are due to the substrate peaks. 


\subsection{In-plane measurements}

The in-plane measurements were carried out for a $>180 \mathrm{~nm}$ thick film grown on each substrate. The grazing incident angle $(\omega)$ was set to $0.4^{\circ}$ for all scans, and the exit angle $(2 \theta)$ varied to optimised the intensity of the film peak. The film peaks were fitted using a pseudo-Voigt functions in order to calculate the lattice parameters.

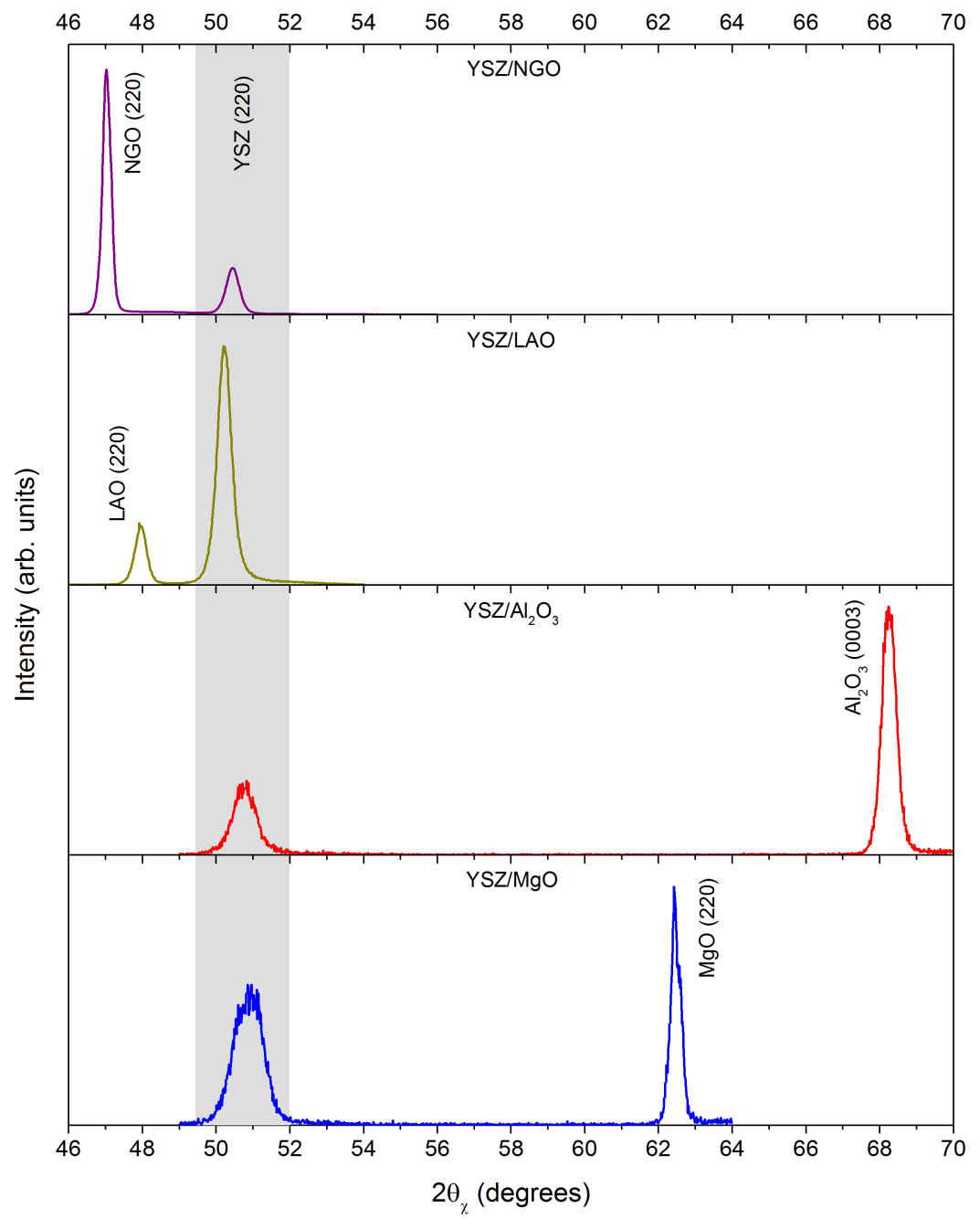

Figure 7: XRD $2 \theta_{\chi}$ patterns from $>180 \mathrm{~nm}$ thick films grown on each substrate. 


\section{TEM film characterisation}

Bright field TEM was performed on the $>180 \mathrm{~nm}$ thick films grown on each substrate to highlight the microstructure. The micrographs of the films grown on $\mathrm{MgO}, \mathrm{LAO}$ and NGO are shown in Fig. 8, and show a similar columnar microstructure as the film grown on $\mathrm{Al}_{2} \mathrm{O}_{3}$ as shown in the article. The columnar structure was not visible for the film grown on LAO, however this is attributed to amorphous damage induced by the FIB section preparation process, which prevents a clear image of the grain structure.
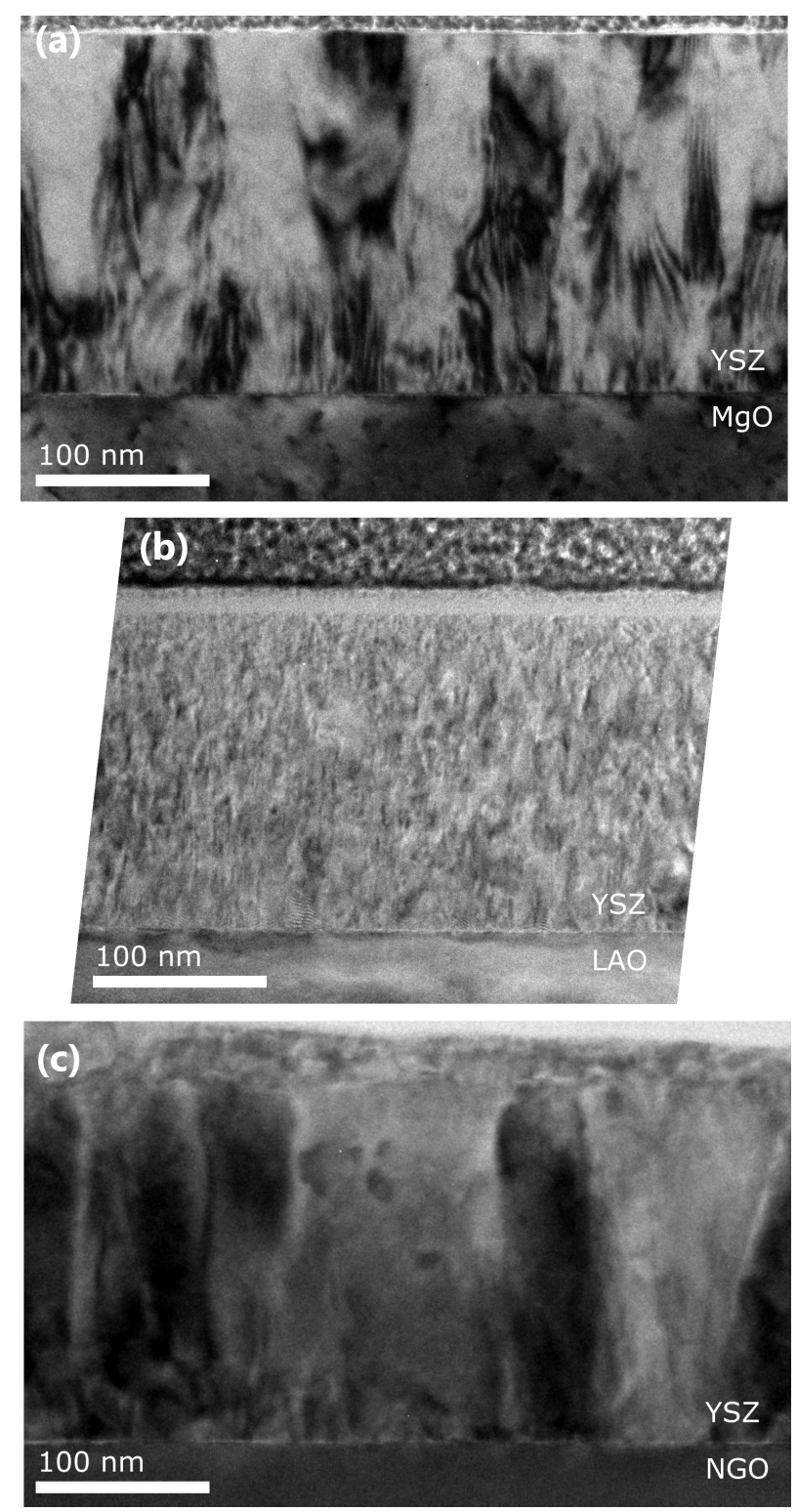

Figure 8: Bright field images of $>180 \mathrm{~nm}$ films grown on (a) $\mathrm{MgO}$, (b) LAO, and (c) NGO. 


\section{Electrochemical impedance spectroscopy}

The in-plane conductivity of the samples was measured by electrochemical impedance spectroscopy (EIS), using an electrode geometry as shown in Fig. 9a. In this geometry, one must take into consideration the capacitance induced by the sample geometry, namely the substrate $\left(C_{\text {substrate }}\right)$, and the capacitance coming from the contacting wires $\left(C_{\text {wiring }}\right)$. Therefore, the equivalent circuit for such a measurement is given in Fig. 9b. These additional capacitances can make the separation of the bulk and grain boundary responses impossible for the electrode geometries used [2], and therefore the impedance response always consisted of a single arc, which was interpreted using the equivalent circuit in Fig. 9c.

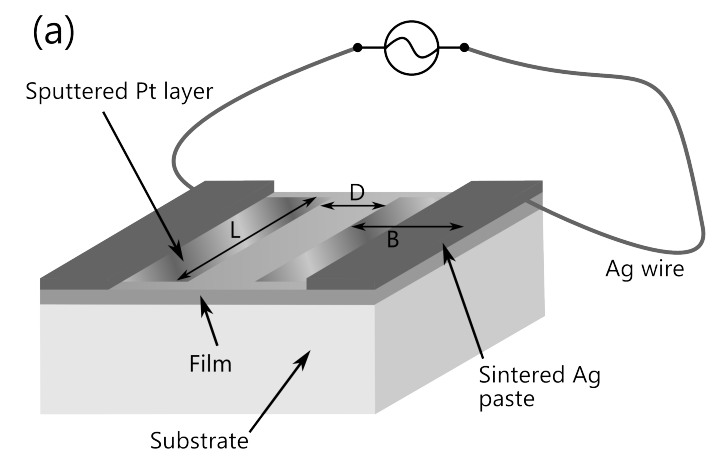

(b)

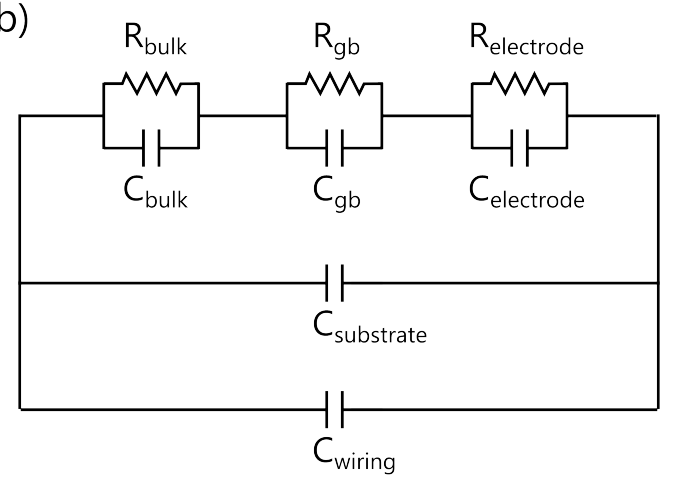

(c)

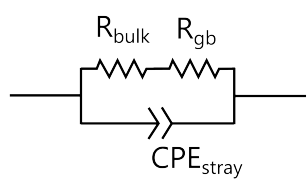

Figure 9: (a) schematic of the electrode geometry used. (b) the equivalent circuit for a thin polycrystalline film of an ionic conductor on an insulating substrate. (c) the modified equivalent circuit used to fit the Nyquist plots in this work.

To ensure that substrates did not contribute to the conductance measured in the samples, as-received substrates were measured using the same electrode geometry as used for the films (Fig. 9a). The conductance of the $\mathrm{MgO}$ and $\mathrm{Al}_{2} \mathrm{O}_{3}$ substrates were found to be approximately two orders of magnitude lower than the thinnest YSZ films measured for the whole temperature range. However, both the LAO and the NGO substrates displayed conductances higher than even a $185 \mathrm{~nm}$ thick YSZ film as shown in Fig. 10. Hence, films grown on LAO and NGO were not assessed by EIS, as electrical leakage through the substrates would dominate the electrical 
response.

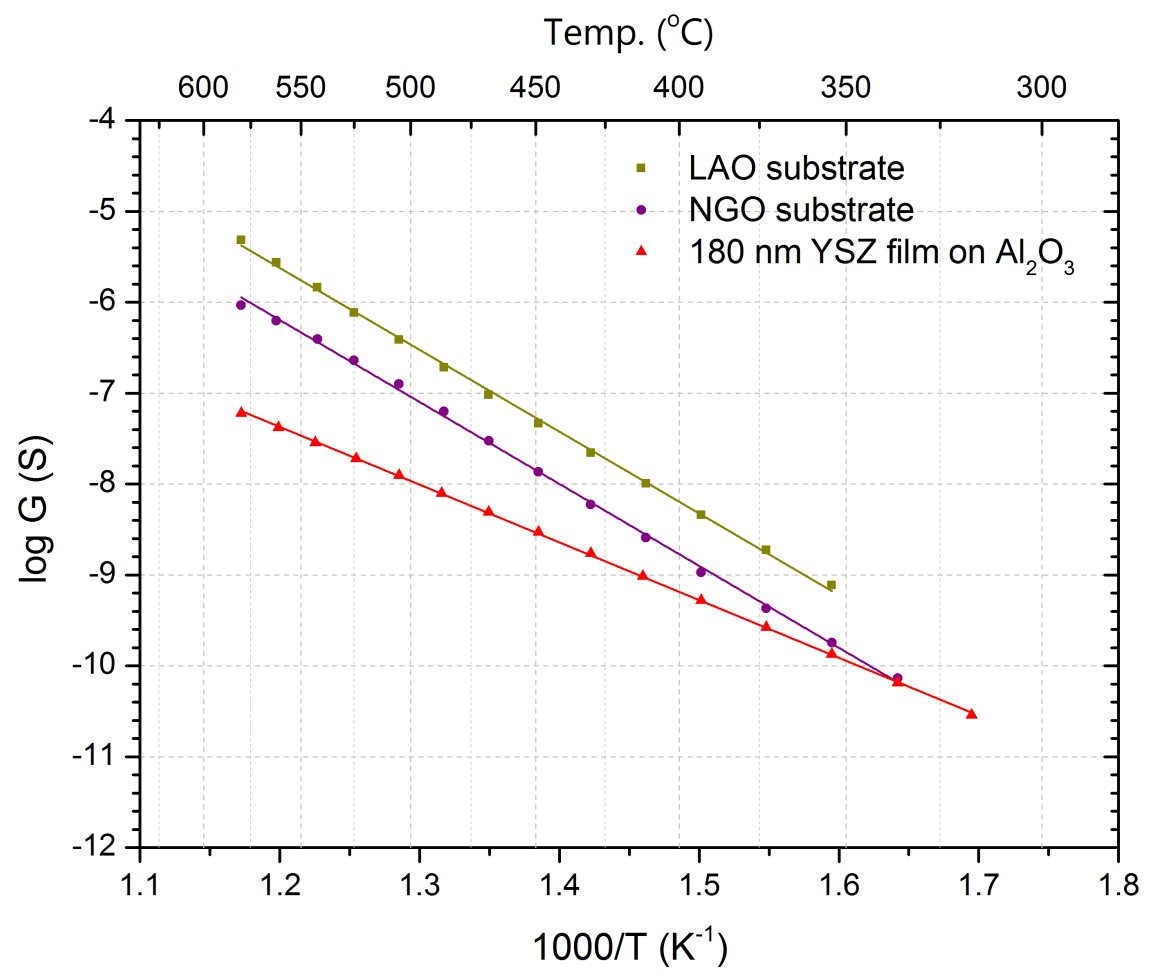

Figure 10: Conduction of a LAO and NGO substrate compared to a $185 \mathrm{~nm}$ thick YSZ film grown on $\mathrm{Al}_{2} \mathrm{O}_{3}$ using the same electrode geometry. 


\section{$5 \quad$ Isotope exchange diffusion profiling}

A TOF-SIMS (TOF.SIMS ${ }^{5}$, ION-TOF Gmbh, Germany) was employed in the 'burst alignment' mode, using a pulsed $25 \mathrm{keV} \mathrm{Bi}^{+}$primary analysis beam, a high current $2 \mathrm{keV} \mathrm{Cs}{ }^{+}$sputter gun, and a low energy $(\sim 20 \mathrm{eV}$ electron gun for charge compensation. To assess the lateral distribution of the ${ }^{18} \mathrm{O}$ tracer, maps were obtained using an analysis area of either $300 \times 300 \mu \mathrm{m}$ or $500 \times 500 \mu \mathrm{m}$, centred in a sputter crater of either $500 \times 500 \mu \mathrm{m}$ or $800 \times 800 \mu \mathrm{m}$ respectively. All maps taken in this work consisted of $512 \times 512$ pixels.

The incorporation and diffusion of an oxygen tracer gas into the films was achieved using a custom made set-up. The films were annealed in isotopically ${ }^{18} \mathrm{O}$-enriched gas inside a quartz tube, which had previously been evacuated to a pressure of $<5 \times 10^{-7}$ mbar. The pressure of the ${ }^{18} \mathrm{O}$ gas was kept to $200 \mathrm{mbar}$ for all experiments. A furnace suspended above the tube was pre-heated to the desired temperature and then lowered onto the tube, after which the sample holder was immediately moved within the tube to the hot-zone. The temperature of the samples was monitored using a thermocouple adjacent to the sample. Once the sample had been annealed for the desired time, it was removed from the hot-zone of the tube and the furnace raised off the tube. This facilitated rapid heating and cooling rates, allowing a well-defined anneal time. The effective anneal time and temperature were corrected according to the method introduced by Killoran [3], as discussed by De Souza et al. [4] for this application. Once the ${ }^{18} \mathrm{O}$ enriched gas had cooled, a residual gas analyser (RGA) was used to check the concentration of the enriched gas which was $>90 \%$ for all exchange anneals.

Isotope exchange diffusion profiling experiments typically involve fitting the diffusion profile using an analytical solution of Ficks second law of diffusion using a set of boundary conditions dictated by the geometry of the sample and parameters of the experiment.

$$
\frac{\delta C}{\delta t}=D \frac{\delta^{2} C}{\delta x^{2}}
$$

Here, $C$ is the concentration of the diffusing substance, $x$ the space coordinate measured normal to the section, and $D$ the diffusion constant. In this work, a two-step diffusion method has been applied similar to that demonstrated by Gerstl et al. [5]. This involves creating a step function in the normalised concentration of the ${ }^{18} \mathrm{O}$ isotope laterally in the film, such that the boundary conditions and solution to Eq. 1 are given by Eqs. 2. 
Boundary Conditions:

At $t=0$ :

$C=C_{0}, x<0$,

$C=C_{b g}, x>0$,

\section{Solution:}

$$
\begin{array}{r}
C(x, t)= \\
=\frac{C_{0}-C_{b g}}{2} \operatorname{erfc}\left(\frac{x}{2 \sqrt{D^{*} t}}\right)+C_{b g}
\end{array}
$$
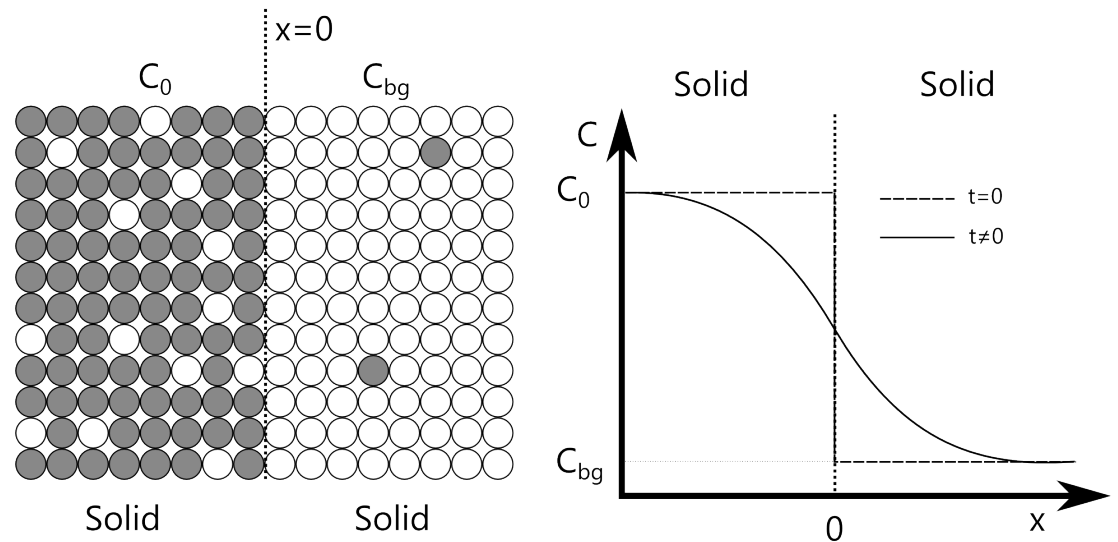

A step function in the normalised concentration of the ${ }^{18} \mathrm{O}$ isotope was created by covering part of the film in a Au blocking layer and subjecting it to a low temperature $\left(300^{\circ} \mathrm{C}\right)$ anneal in ${ }^{18} \mathrm{O}$ enriched gas for 30 mins, referred to as the incorporation step. The Au layers were approximately $100 \mathrm{~nm}$ thick and were deposited at room temperature using a magnetron sputter coater (K575X, Emitech Ltd, UK or Q150T, Quorum Technologies Ltd, UK). The Au layer was then removed and the ${ }^{18} \mathrm{O}$ isotope distribution mapped using SIMS to ensure the boundary conditions applied. Next another Au blocking layer was deposited onto the film, this time covering the entire surface such that oxygen could not move in or out of the YSZ. This was then annealed in the same furnace, but under vacuum and at $500^{\circ} \mathrm{C}$, referred to as the diffusion step. Finally the $\mathrm{Au}$ layer was removed again and the ${ }^{18} \mathrm{O}$ concentration mapped by SIMS. The second SIMS analysis was always obtained in a different, but equivalent, area to first so that any beam damage induced by the first SIMS measurement did not affect the observed diffusion. 

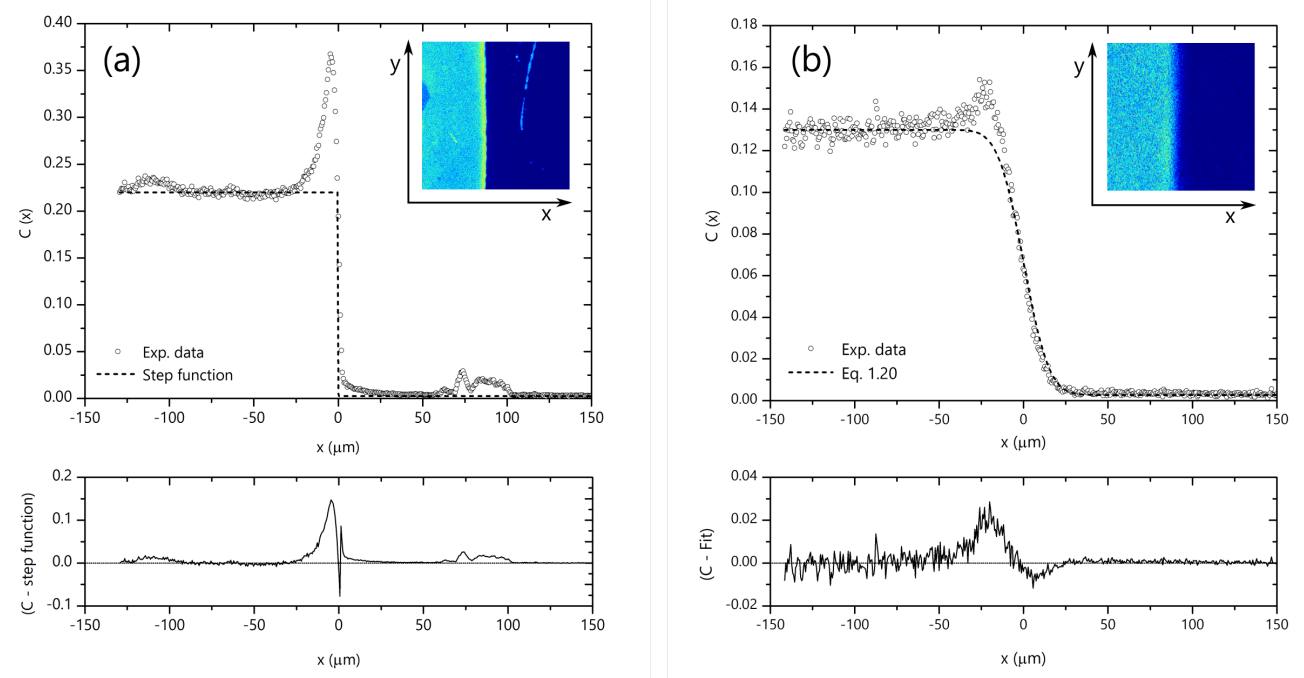

Figure 11: (a) normalised ${ }^{18} \mathrm{O}$ ratio after the incorporation step, compared to a step function. (b) the normalised ${ }^{18} \mathrm{O}$ ratio after the diffusion step, fitted using Eq. 2. The insets show the maps of the ${ }^{18} \mathrm{O}$ normalised concentration. Below are the differences between the real and idealised cases.

Typical resultant ${ }^{18} \mathrm{O}$ profiles after the incorporation step, and the diffusion step are shown in Fig. 11a and Fig. 11b respectively. Commonly a sizable decrease in the ${ }^{18} \mathrm{O}$ concentration was observed after the diffusion step. The most likely cause for this would be diffusion into the substrate. Although nominally ionically insulating, only a short diffusion length into the substrate would still significantly deplete the limited reservoir in the film.

Also for all samples a spike in the ${ }^{18} \mathrm{O}$ concentration at $x=0$ is observed. Although this was initially surprising, a closer look at the Au edge after the incorporation step may provide an explanation for the increase in the ${ }^{18} \mathrm{O}$ concentration, as shown in the SEM images in Fig. 12. Although there is no noticeable roughness of the interface over scales of several hundreds of $\mu \mathrm{m}$ (typical area size of SIMS map obtained), the Au was found to ball up at the edge over an approximately $2 \mu \mathrm{m}$ region, forming particles ranging from $\sim 100 \mathrm{~nm}$ to $<50 \mathrm{~nm}$. This region most likely occurs due to the Au layer thinning towards the edge of the film. This could be due to either shadowing effects from height of the mask, or the mask not lying flat on the film surface. 

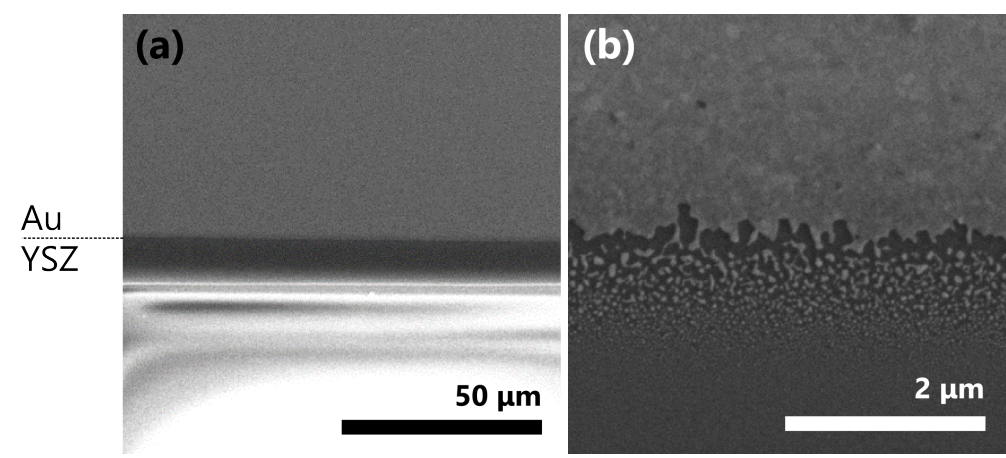

Figure 12: SEM micrographs of the Au edge created using a Omniprobe lift-out grid as a mask.

The observed nanoscopic Au particles like act as catalysts for oxygen exchange. Although Au is commonly thought of as chemically inert, when in the form of nanoparticles it has been shown to be a very active catalyst for the oxygen reduction reaction [6]. Alternatively, it is conceivable that the cause of the ${ }^{18} \mathrm{O}$ profile is due to residual $\mathrm{Au}$ nanoparticles left on the film surface during the SIMS analysis, leading to a change in the surface charging effects. A localised increase in the total ion intensity could lead to an incorrectly high ${ }^{18} \mathrm{O}$ concentration in that area. However, by plotting both the ${ }^{18} \mathrm{O}$ and the ${ }^{16} \mathrm{O}$ profiles as shown in Fig. 13, it can be seen that the ${ }^{16} \mathrm{O}$, signal decreases at the Au interface signalling that the surface exchange is indeed enhanced by the Au nanoparticles.

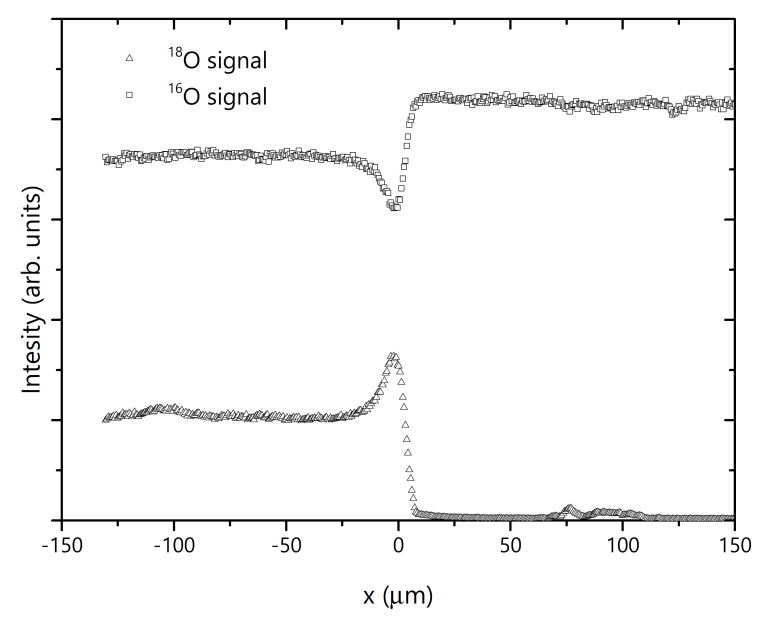

Figure 13: The raw ${ }^{18} \mathrm{O}$ and ${ }^{16} \mathrm{O}$ profiles for the data plotted in Fig. [?]a.

The spike in the normalised ${ }^{18} \mathrm{O}$ concentration was observed for the majority of films measured using this method, however the size and shape of the enriched region was not consistent and varied for all specimen. Clearly the step function is not realised after the incorporation step, and therefore the diffusion profile in Fig. 11b, cannot be accurately described by Eq. 2 . 


\subsection{Numerical Fitting.}

By making the assumption that the areas measured after the incorporation step and diffusion step were equivalent after the incorporation step (i.e. that the Au interface is uniform along each edge), the initial profile may be used as the starting ${ }^{18} \mathrm{O}$ distribution and the final profile fitted numerically. Fig. 14 shows a concentration profile, divided into segments of width $h$, with $C_{1}$, $C_{2}$ and $C_{3}$ being the concentration at the neighbouring interfaces. The lines at $R$ and $S$, denotes the midsections of the two adjacent layers.

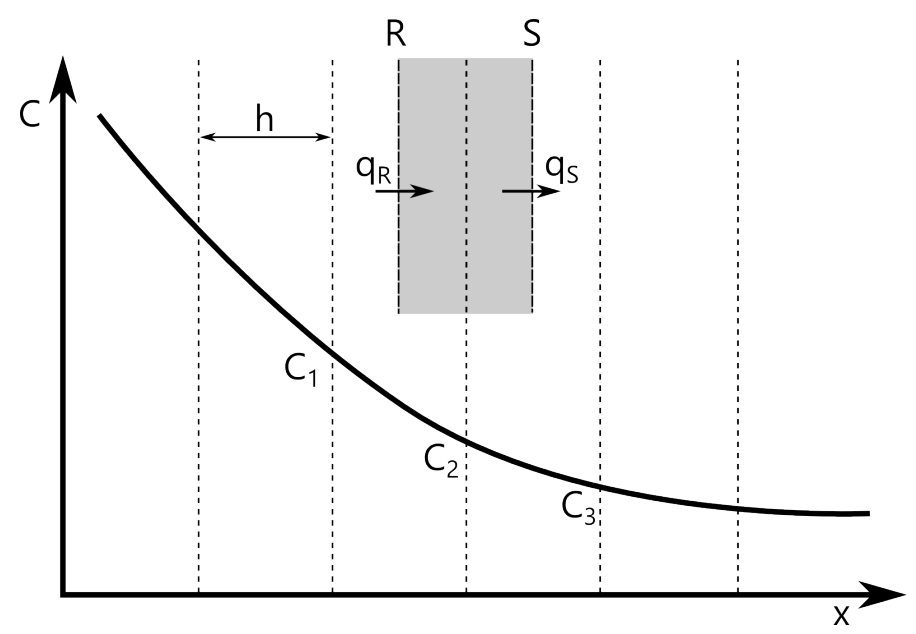

Figure 14: Concentration gradient as a function of $x$.

Fick's first law of diffusion relates the rate of transfer of a diffusant per unit area of a section, $F$, to the concentration of the diffusing substance, $C$, the space coordinate measured normal to the section, $x$, and a diffusion constant, $D$.

$$
F=-D \frac{\delta C}{\delta x}
$$

Hence it may be shown that in a short time, $\tau$, the amount of diffusant entering the shaded region through the unit surface area $R$ is given by [7]

$$
q_{R}=-\frac{D \tau}{h}\left(C_{2}-C_{1}\right)
$$

and similarly, the amount of diffusant leaving the shaded region through the unit surface $S$ is given by

$$
q_{S}=-\frac{D \tau}{h}\left(C_{3}-C_{2}\right) .
$$

Hence the net amount of diffusant accumulating in the shaded region is

$$
q_{R}-q_{S}=-\frac{D \tau}{h}\left(C_{2}-C_{1}-C_{3}+C_{2}\right)=\frac{D \tau}{h}\left(C_{1}-2 C_{2}+C_{3}\right) .
$$

Therefore, by taking $C_{2}$ to represent the average concentration of the narrow shaded region, the net change in the concentration of the segment can be approximated to

$$
C_{2}^{\prime}-C_{2}=\frac{D \tau}{h^{2}}\left(C_{1}-2 C_{2}+C_{3}\right)
$$


where $C_{2}^{\prime}$ is the concentration at the end of the interval $\tau$.

These calculations was performed in the MATLAB software using a home-written script. The profiles after the diffusion step were simulated for a range of $D$ values, using the initial profiles as the starting concentration and the time of the diffusion step, $t_{\text {diff }}$. The goodness of fit was calculated using a $\chi^{2}$ function, and the $D$ values optimised to three significant figures. In addition to $D$ as a fitting parameter, the simulated profiles were free to translate along the $x$ direction to account for an offset in the centre position of the Au edge, and to multiply the entire set of $y$ values by a coefficient to account for systematic errors in the calculated ${ }^{18} \mathrm{O}$ fraction and diffusion into the substrate between the initial and final profiles.

The width $h$, was taken as the width of a pixel. The time-constant, $\tau$, needs to be sufficiently small to accurately simulate the resultant diffusion profile, however as $\tau$ is decreased the length of the calculation increases rapidly. Therefore, in order to optimise the $\tau$ values used, a simulated profile according to Eq. $2\left(D^{*}=1.00 \times 10^{-9} \mathrm{~cm}^{2} \mathrm{~s}^{-1}, t_{\text {diff }}=5000 \mathrm{~s}, h=0.98 \mu \mathrm{m}\right)$ was fitted numerically using a range of $\tau$ values. The numerically fitted $D^{*}$ values are shown in Fig. 15, along with the relative error. From this it can be seen that there is minimal difference for $\tau \leq 0.05$ $\mathrm{s}$, and the error is close to $\sim 2 \%$. Hence a value of $\tau=0.05 \mathrm{~s}$ is used in all the numerical fitting in this work, such that the calculations can be performed on acceptable time scales.

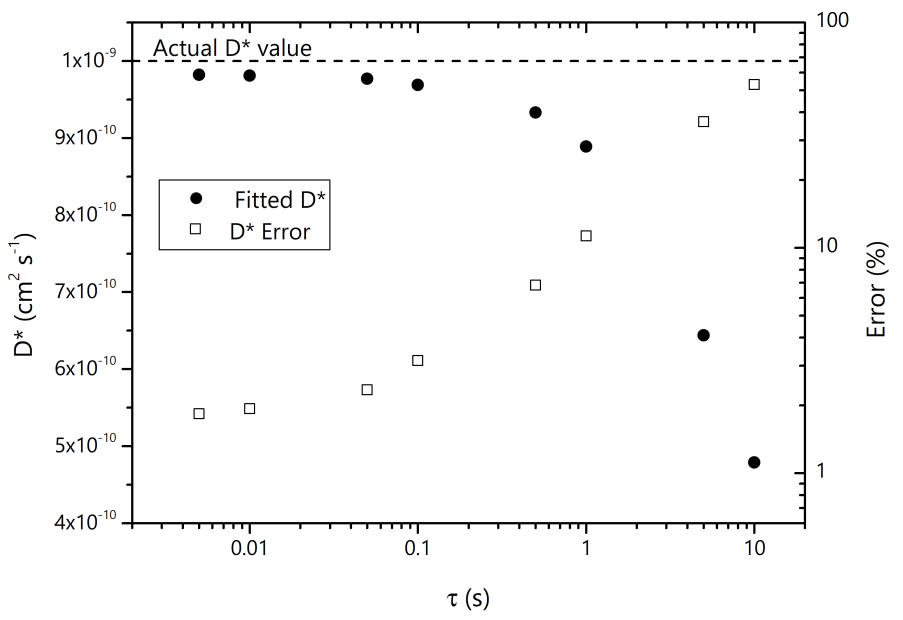

Figure 15: The effect of the time constant, $\tau$, on the $\mathrm{D} *$ values and relative errors of a numerically fitted profile which was simulated according to Eq. 2 with a $D^{*}$ of $1.00 \times 10^{-9}$.

Fig. 16 shows the same profile plotted in Fig. 11b, here fitted numerically. The enriched area in the profile, occurring at the position of the Au interface is reproduced by the fitting procedure. The difference between the experimental data and the fit is plotted below, which is significantly improved compared to fitting by the empirical solution (Eq. 2) as in Fig. 11b. The poor agreement between the fitted and experimental profile around the $x \sim 25$ and $x \sim 225$ regions are due to artefacts in the initial profile as seen in Fig. 11a, are most likely originate from an imperfect Au blocking layer. However these regions are not included when fitting the data to obtain a $D^{*}$ value.

The two step diffusion method, could be further optimised by extending the diffusion time, $t_{d i f f}$, from 2 hours (as it was in Figs. 11 and 16) to 14 hours, thereby lengthening the profile and 
reducing the error due to the focus of the SIMS analysis beam. As seen in Section 5.2, this also resulted in profiles similar in shape to Eq. 2 in some cases, reducing the sensitivity of the quality of fit on the initial profile after the incorporation step. This is beneficial, if there is a difference in the Au edge between the analysis areas used to obtain the profiles following the incorporation and diffusion steps.
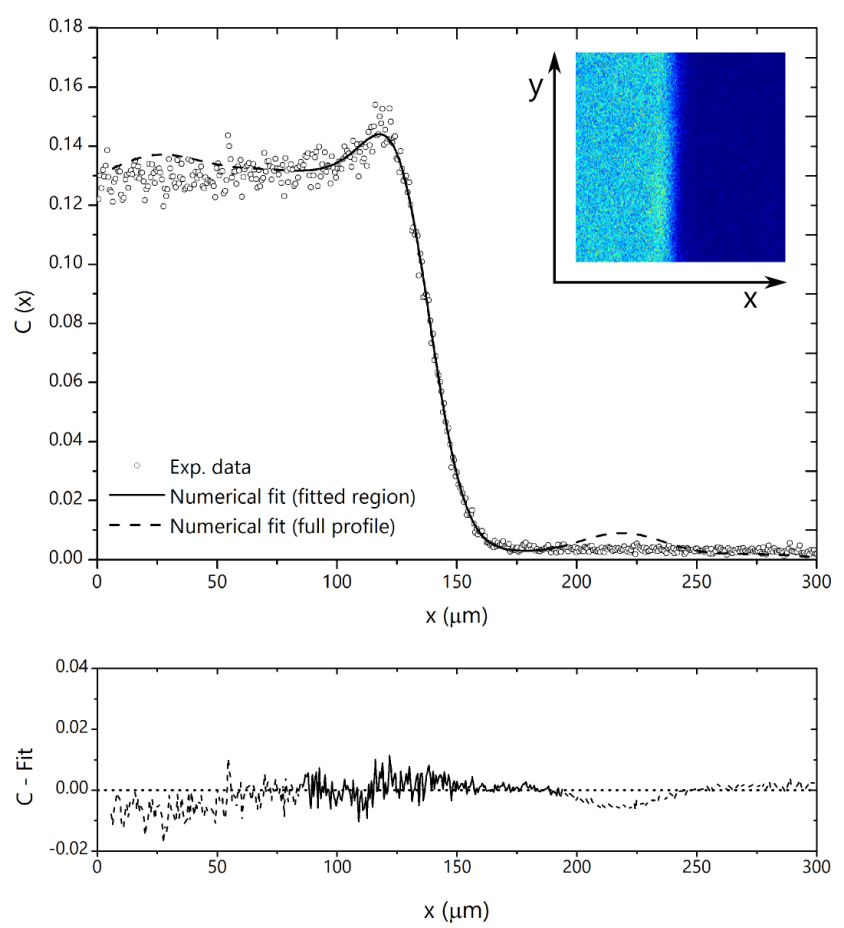

Figure 16: Normalised ${ }^{18} \mathrm{O}$ ratio after the diffusion step, fitted using Eq. 2. The insets show the maps of the ${ }^{18} \mathrm{O}$ normalised concentration. Below is the differences between the experimental data and the fit. The solid lines represent the region fitted using the $\chi^{2}$ function, and the dashed shows the rest of the simulated profile for the final $D^{*}$ value. Note that the $x$-axis is no longer centred at $x=0$, as it was in Figs. 11a and 11b, because this is no longer required for the numerical fitting procedure as it was for when fitting using the empirical solution Eq. 2. 


\subsection{Diffusion profiles}
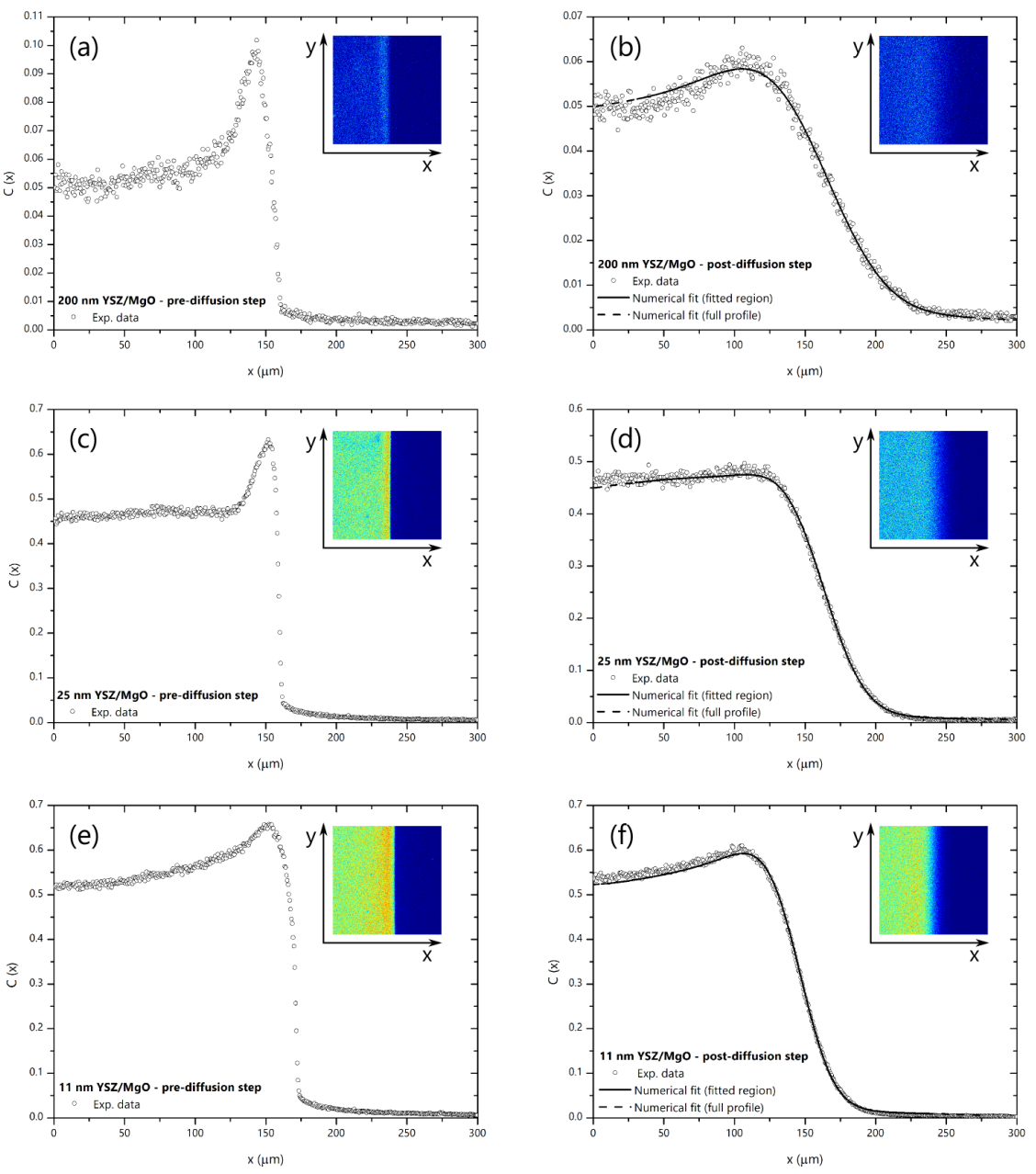

Figure 17: Lateral profiles of the ${ }^{18} \mathrm{O}$ isotope fraction for YSZ films grown on $\mathrm{MgO}$. The insets show the ${ }^{18} \mathrm{O}$ isotope fraction integrated in the $z$-axis direction. In (a, c, and e) the ${ }^{18} \mathrm{O}$ distribution across the $\mathrm{Au}$ covered and uncovered region is shown after the incorporation step of 30 minutes at $300^{\circ} \mathrm{C}$ in ${ }^{18} \mathrm{O}$ enriched gas. In (b, d, and $\mathrm{f}$ ) the subsequent ${ }^{18} \mathrm{O}$ distribution after annealing for 14 hours at $500^{\circ} \mathrm{C}$ is shown. The profiles in (b, d, and f) are fitted using the numerical method in Section 5.1. 

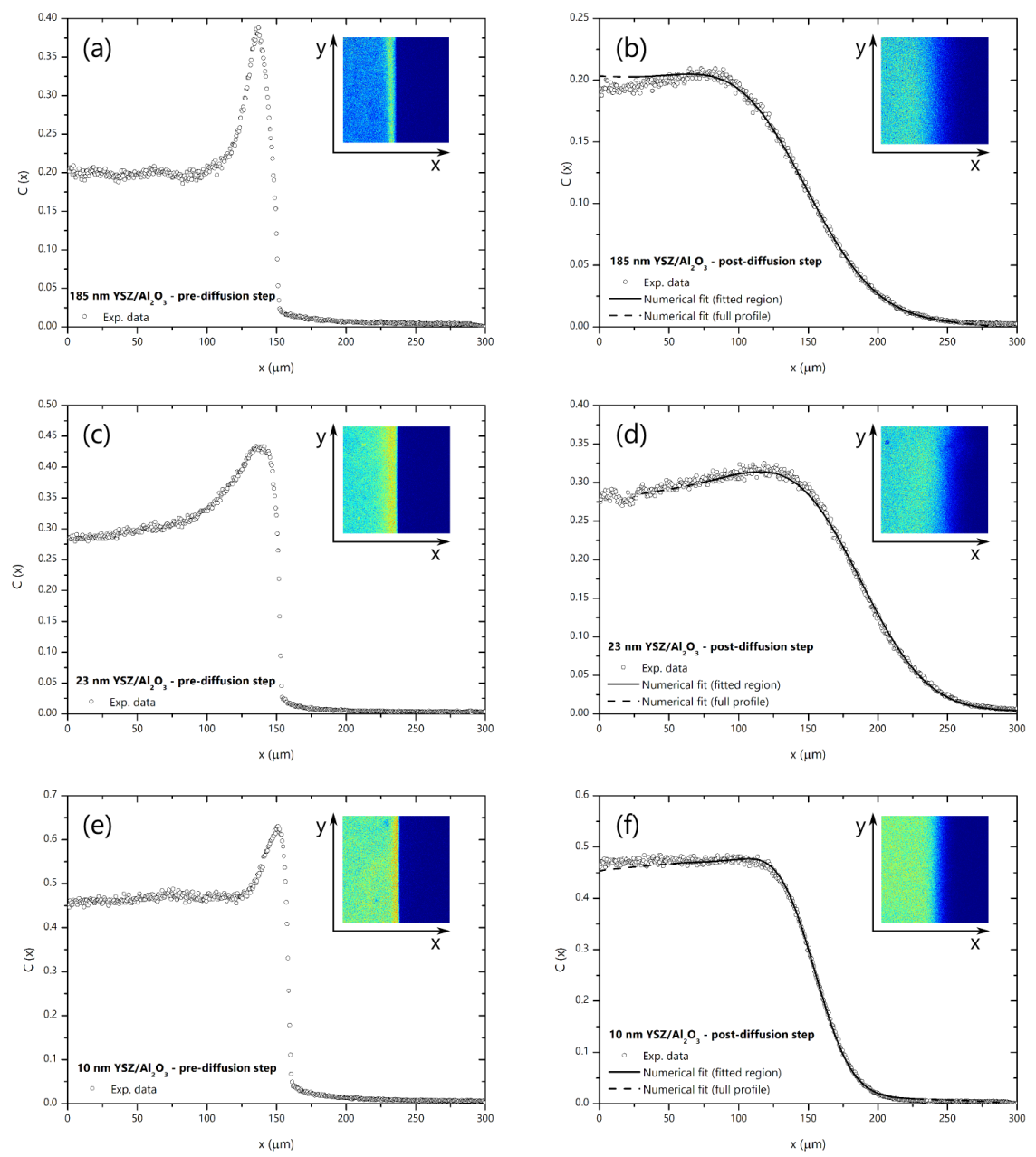

Figure 18: Lateral profiles of the ${ }^{18} \mathrm{O}$ isotope fraction for YSZ films grown on $\mathrm{Al}_{2} \mathrm{O}_{3}$. The insets show the ${ }^{18} \mathrm{O}$ isotope fraction integrated in the $z$-axis direction. In $\left(\mathrm{a}, \mathrm{c}\right.$, and e) the ${ }^{18} \mathrm{O}$ distribution across the $\mathrm{Au}$ covered and uncovered region is shown after the incorporation step of 30 minutes at $300^{\circ} \mathrm{C}$ in ${ }^{18} \mathrm{O}$ enriched gas. In (b, d, and $\mathrm{f}$ ) the subsequent ${ }^{18} \mathrm{O}$ distribution after annealing for 14 hours at $500^{\circ} \mathrm{C}$ is shown. The profiles in (b, d, and f) are fitted using the numerical method in Section 5.1. 

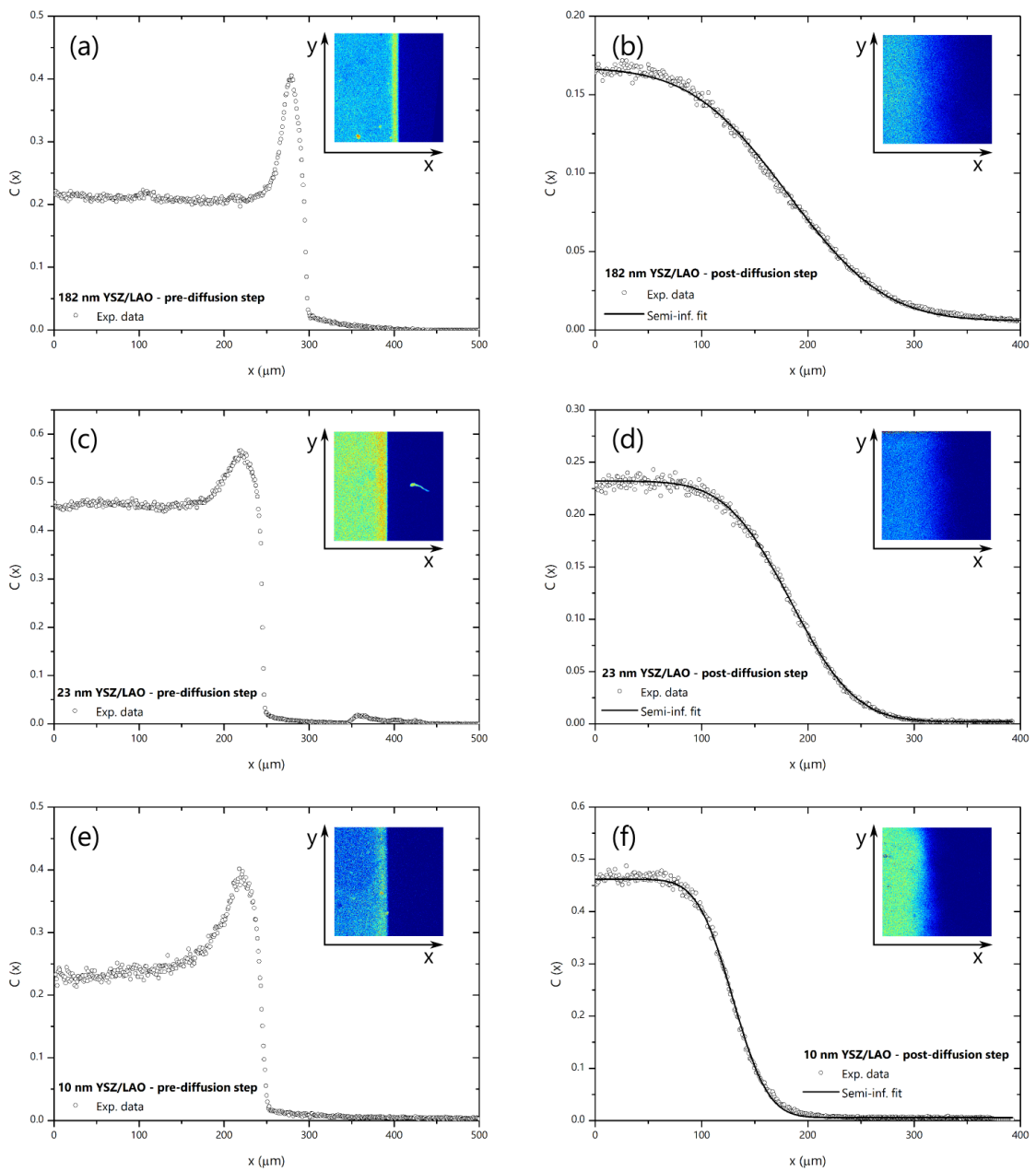

Figure 19: Lateral profiles of the ${ }^{18} \mathrm{O}$ isotope fraction for YSZ films grown on LAO. The insets show the ${ }^{18} \mathrm{O}$ isotope fraction integrated in the $z$-axis direction. In (a, c, and e) the ${ }^{18} \mathrm{O}$ distribution across the $\mathrm{Au}$ covered and uncovered region is shown after the incorporation step of 30 minutes at $300^{\circ} \mathrm{C}$ in ${ }^{18} \mathrm{O}$ enriched gas. In (b, d, and $\mathrm{f}$ ) the subsequent ${ }^{18} \mathrm{O}$ distribution after annealing for 14 hours at $500^{\circ} \mathrm{C}$ is shown. The profiles in (b, d, and f) are fitted according to Eq. 2. 

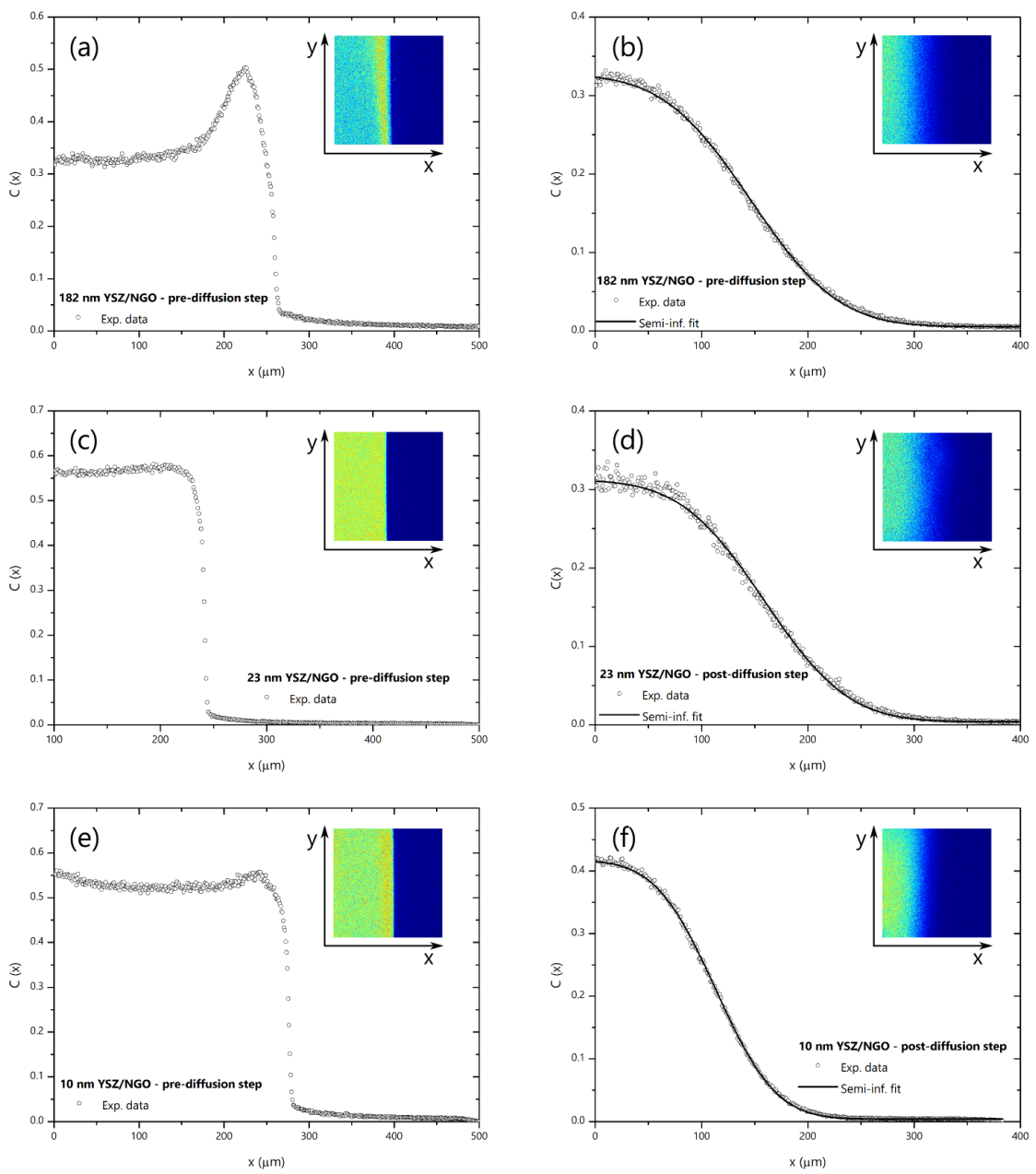

Figure 20: Lateral profiles of the ${ }^{18} \mathrm{O}$ isotope fraction for YSZ films grown on NGO. The insets show the ${ }^{18} \mathrm{O}$ isotope fraction integrated in the $z$-axis direction. In (a, c, and e) the ${ }^{18} \mathrm{O}$ distribution across the $\mathrm{Au}$ covered and uncovered region is shown after the incorporation step of 30 minutes at $300^{\circ} \mathrm{C}$ in ${ }^{18} \mathrm{O}$ enriched gas. In (b, d, and $\mathrm{f}$ ) the subsequent ${ }^{18} \mathrm{O}$ distribution after annealing for 14 hours at $500^{\circ} \mathrm{C}$ is shown. The profiles in (b, d, and $\mathrm{f}$ ) are fitted according to Eq. 2. 
6 Structure and transport values of the YSZ films $\equiv$ 


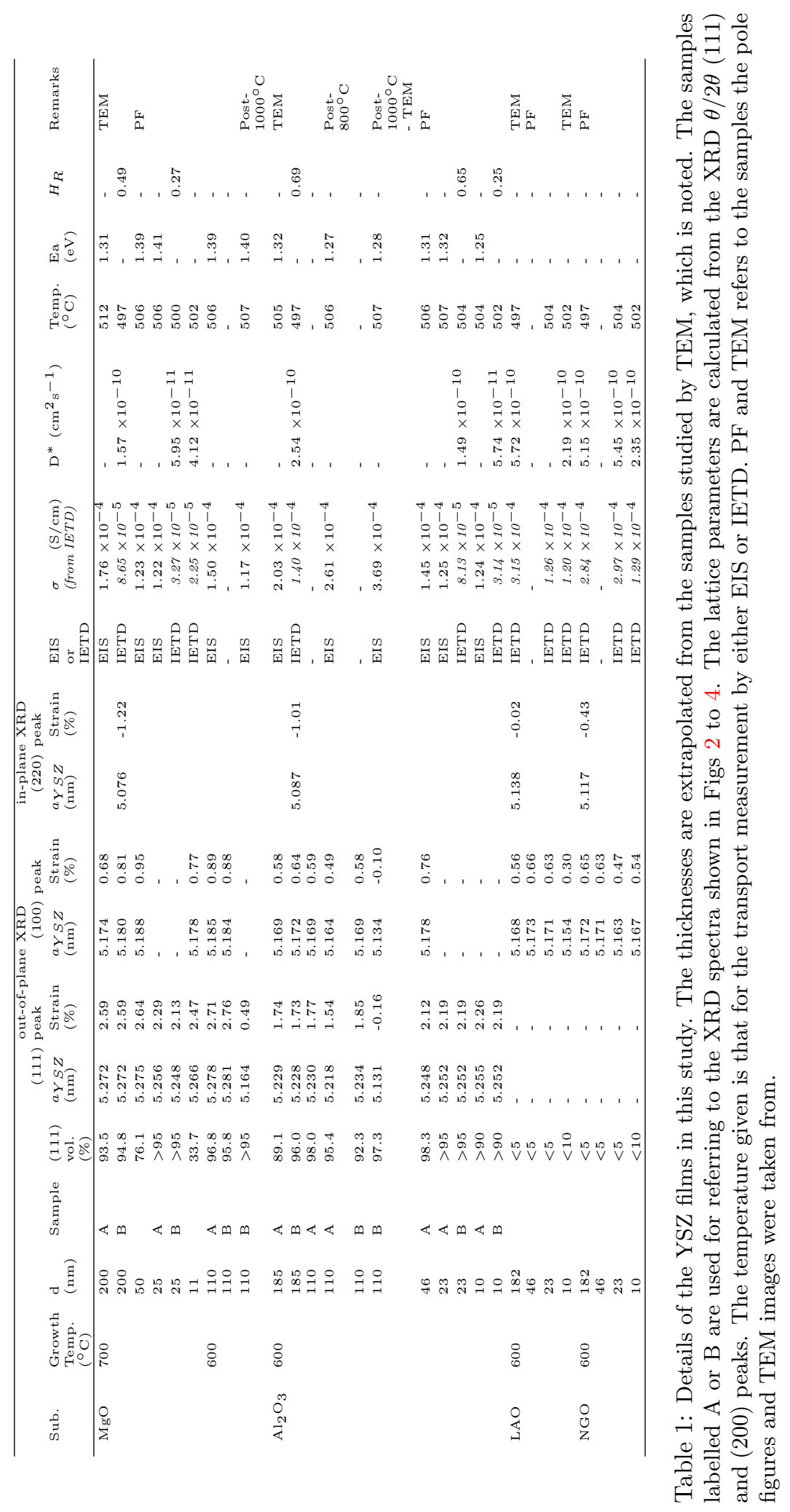




\section{Calculating the substrate mismatch}

From the orientation relationships obtained by the XRD and SAED analysis and from the density of interfacial dislocations as observed by HR-TEM, the strain present at the interfaces can, in principal, be calculated for the YSZ films grown on each substrate. The lattice parameter of the YSZ is taken from the sintered PLD target $(0.5139 \mathrm{~nm}$, see Fig. 1), and the substrate lattice parameters are taken from the literature and are given in Table 2 of the article.

The dominant orientation relationship for the YSZ films grown on $\mathrm{MgO}$ is given by:

$$
\text { YSZ (111)||MgO (001) }
$$

In plane, four discrete orientations were observed, each related by either a $90^{\circ}$ or a $180^{\circ}$ rotation in the azimuth. From the relationships given in the article it can be seen that for each orientation the family of YSZ (112) planes is parallel to the family of MgO (110) planes. The atomic spacing of the close packed Zr cations is equal to $3 / 2$ of the lattice spacing, $d_{Y S Z(112)}$, of the YSZ (112) planes. For $\mathrm{MgO}$ the atomic spacing of the close packed $\mathrm{O}$ anions is equal to the (110) spacing, $d_{M g O(110)}$. The lattice mismatch in this direction can be calculated as

$$
f_{M g O(110), Y S Z(112)}=\frac{d_{M g O(110)}-\frac{3}{2} d_{Y S Z(112)}}{\frac{3}{2} d_{Y S Z(112)}}=-5.34 \%
$$

Due to the different symmetries of the YSZ (111) and MgO (100) planes, the lattice mismatch is different for the two in-plane perpendicular directions. For the YSZ (110) planes parallel to the $\mathrm{MgO}$ (110) planes, the Zr spacing is equal to the lattice spacing, $d_{Y S Z(110)}$, of the YSZ (110) planes, and so

$$
f_{M g O(110), Y S Z(110)}=\frac{d_{M g O(110)}-d_{Y S Z(110)}}{d_{Y S Z(110)}}=-17.8 \% .
$$

These are equivalent for each of the in-plane orientations of the YSZ grains. Such a large lattice mismatch would almost certainly result in dislocations forming along the interface, however these could not be observed by TEM. Hence the values here represent the upper limit of the strain occurring at the substrate-film interface.

For the YSZ films grown on $\mathrm{Al}_{2} \mathrm{O}_{3}$, the dominant out-of-plane orientation is given by:

$$
\text { YSZ (111) }|| \mathrm{Al}_{2} \mathrm{O}_{3} \text { (0001) }
$$

From the XRD pole figures and SAED patterns, it was shown that two in plane orientations existed for the grains, each related by a rotation of $60^{\circ}$ or a mirror inversion. From the relationships given the article, one may see that for the two orientations the YSZ (110) planes are aligned along the $\mathrm{Al}_{2} \mathrm{O}_{3}$ (1100) planes. As before, the spacing of the $\mathrm{Zr}$ cations are equal to the lattice spacing, $d_{Y S Z(110)}$, and the close packed spacing of the $\mathrm{O}$ anions is equal to $1 / 3$ of the lattice spacing, $d_{\mathrm{Al}_{2} \mathrm{O}_{3}(\overline{1} 100)}$, in $\mathrm{Al}_{2} \mathrm{O}_{3}$. From the HR-TEM analysis, a dislocation occurs every 4 or 5 columns $\left(1 / 2\right.$ of $\left.d_{A_{2} \mathrm{O}_{3}(1100)}\right)$, therefore between a $4: 5$ and $3: 4$ commensurability.

$$
f_{A l_{2} O_{3}(\overline{1} 100), Y S Z(110)}=\frac{4(5) \frac{1}{3} d_{A l_{2} O_{3}(\overline{1} 100)} 3(4) d_{Y S Z(110)}}{3(4) d_{Y S Z(110)}}=0.703(-5.59) \% .
$$

Hence, depending on the exact density of the dislocation network, the lattice mismatch is between +0.703 and $-5.59 \%$. In the perpendicular direction, The atomic spacing of the close packed $\mathrm{O}$ anions is equal to the lattice spacing $d_{A_{2} O_{3}(\overline{11} 20)}$, in $\mathrm{Al}_{2} \mathrm{O}_{3}$, however no information on the dislocation density could be obtained from the TEM analysis.

$$
f_{A l_{2} O_{3}(\overline{11} 20), Y S Z(112)}=\frac{\frac{3}{2} d_{A l_{2} O_{3}(\overline{11} 20)} \frac{3}{2} d_{Y S Z(112)}}{\frac{3}{2} d_{Y S Z(112)}}=24.47 \% .
$$


As with the YSZ/MgO interface, this represents the upper limit of the interfacial strain.

From the XRD analysis and SAED the out-of-plane orientation for the YSZ films grown on LAO substrates was confirmed to be:

$$
\text { YSZ (100)||LAO (100) }
$$

For YSZ grown on LAO, a single in-plane orientation was observed. LAO adopts the perovskite crystal structure, with a slight distortion from the cubic symmetry $\alpha=90.096$, however this is expected to have little impact here and the structure is treated as cubic. The surface termination of the perovskite oxides is commonly thought to be $\mathrm{AO}$ plane terminated [8]. For an $\mathrm{AO}$ surface the atomic spacing between the atomic $\mathrm{O}$ anions is equal to the LAO (001) lattice spacing, $d_{L A O(001)}$, along the (001) plane. The spacing of the Zr cations on the YSZ (110) plane is equal to the lattice spacing, $d_{Y S Z(110)}$. From the HR-TEM imaging, a dislocation was found to occur on average every 27 YSZ (220) planes. Hence assuming a 27:26 commensurability the lattice mismatch is calculated as:

$$
f_{L A O(001), Y S Z(110)}=\frac{26 d_{L A O(001)}-27 d_{Y S Z(110)}}{27 d_{Y S Z(110)}}=0.44 \% .
$$

For the YSZ films grown on NGO substrates the following orientation were observed by XRD:

$$
\text { YSZ (100)||NGO (110) }
$$

NGO is a perovskite material which adopts an orthorhombic crystal structure. A single inplane orientation was found according to the analysis from pole figures and SAED. The spacing between the $\mathrm{O}$ anions in the NGO [001] direction is equal to the NGO (001) spacing, and the NGO (110) spacing in the [010] direction. The dislocation density from the TEM was found to occur on average every 16.6 YSZ (220) planes. Therefore assuming the density of dislocations are the same in both in-plane directions, and a commensurability of 83:78 the lattice mismatch is given by

$$
\begin{aligned}
& f_{N G O(001), Y S Z(011)}=\frac{78 d_{N G O(001)}-83 d_{Y S Z(011)}}{83 d_{Y S Z(011)}}=0.13 \% . \\
& f_{N G O(110), Y S Z(011)}=\frac{78 d_{N G O(110)}-83 d_{Y S Z(011)}}{83 d_{Y S Z(011)}}=0.15 \% .
\end{aligned}
$$

\section{References}

[1] FullProf Suite. Version 5.60 - Jan2015-ILL JRC. 2015.

[2] M. Gerstl, E. Navickas, G. Friedbacher, F. Kubel, M. Ahrens, and J. Fleig. The separation of grain and grain boundary impedance in thin yttria stabilized zirconia (YSZ) layers. Solid State Ionics, 185(1):32-41, 2011.

[3] D. R. Killoran. The Effective Duration of a Linear Slow-Cool. Journal of the Electrochemical Society, 109:107-171, 1963.

[4] R. A. De Souza, J. A. Kilner, and J. F. Walker. A SIMS study of oxygen tracer diffusion and surface exchange in $\mathrm{La}_{0.8} \mathrm{Sr}_{0.2} \mathrm{MnO}_{3+\delta}$. Materials Letters, 43(12):43-52, 2000.

[5] M. Gerstl, G. Friedbacher, F. Kubel, H. Hutter, and J. Fleig. The relevance of interfaces for oxide ion transport in yttria stabilized zirconia (YSZ) thin films. Physical Chemistry Chemical Physics, 15:1097 - 1107, 2013. 
[6] M. Daniel and D. Astruc. Gold nanoparticles: assembly, supramolecular chemistry, quantumsize-related properties, and applications toward biology, catalysis, and nanotechnology. Chemical reviews, 104(1):293-346, 2004.

[7] J. Crank. The Mathematics of Diffusion. Clarendon Press, 1979.

[8] J. Druce, H. Tellez, M. Burriel, M. D. Sharp, L. J. Fawcett, S. N. Cook, D. S. McPhail, T. Ishihara, H. H. Brongersma, and J. A. Kilner. Surface termination and subsurface restructuring of perovskite-based solid oxide electrode materials. Energy $\&$ Environmental Science, 7(11):3593-3599, 2014. 